\title{
A clock transition for a future optical frequency standard with trapped atoms
}

\author{
Irène Courtillot, Audrey Quessada, Richard P. Kovacich, Anders Brusch, \\ Dmitri Kolker, Jean-Jacques Zondy, Giovanni D. Rovera, and Pierre Lemonde* \\ BNM-SYRTE, Observatoire de Paris \\ 61, Avenue de l'observatoire, 75014, Paris, France
}

(Dated: August 25, 2018)

\begin{abstract}
We report the first direct excitation of the strongly forbidden $5 s^{2}{ }^{1} S_{0}-5 s 5 p^{3} P_{0}$ transition in ${ }^{87} \mathrm{Sr}$. Its frequency is $429228004235(20) \mathrm{kHz}$. A resonant laser creates a small leak in a magneto-optical trap (MOT): atoms build up to the metastable ${ }^{3} P_{0}$ state and escape the trapping process, leading to a detectable decrease in the MOT fluorescence. This line has a natural width of $10^{-3} \mathrm{~Hz}$ and can be used for a new generation of optical frequency standards using atoms trapped in a light shift free dipole trap.
\end{abstract}

PACS numbers: 06.30.Ft,32.30.Jc,39.30.+W,32.80.-t

In addition to being at the heart of the international system of units, extreme frequency metrology finds a wide range of applications throughout physics. As an example, tests of the stability of fundamental constants are performed by comparing the frequencies of different atomic transitions 1, 22. These laboratory tests are already competitive with those performed at the cosmological scale 3, 4] and improve with the accuracy of the atomic transitions frequency measurements. Microwave frequency standards such as atomic fountains now have relative accuracies better than $10^{-15}$ with potential improvements down to $10^{-16}[5]$. Going further, however, seems extremely difficult due to the limited quality factor of the atomic resonance in these devices $\left(\sim 10^{10}\right)$. Optical frequency standards exhibit much higher line- $Q$, up to $10^{14}$ as recently demonstrated in Ref. [6]. These devices reach fractional frequency instabilities below $10^{-14}$ over 1 s 7], almost one order of magnitude better than fountains 5]. Their current accuracy lies near 1 part in $10^{14}$ with anticipated large room for improvement 2, 8, 9, 10, 11.

Two different approaches are commonly used to develop optical frequency standards [12]. The first one is based on the spectroscopy of a single trapped ion, the second on the spectroscopy of a large ensemble of free falling neutral atoms. It is a commonly shared opinion that the ion approach may lead to a better ultimate frequency accuracy due to the "perfect" control of the ion motion, while the atomic approach should lead to a better frequency stability thanks to the numerous quantum references contributing to the signal. Recently H. Katori proposed a scheme which would combine the advantages of both approaches [13]. The idea is to trap neutral atoms in the Lamb-Dicke regime in an optical lattice operating at a wavelength where the light-shift of the clock transition vanishes.

H. Katori proposed to use ${ }^{87} \mathrm{Sr}$ probed on the $5 s^{2}{ }^{1} S_{0}-$ $5 s 5 p^{3} P_{0}$ line at $698 \mathrm{~nm}$, which indeed seems an ideal system for the realization of this scheme. This $J=0-J=0$ resonance is only slightly allowed by hyperfine coupling and its metrological properties are exquisite. A natural linewidth of $\sim 1 \mathrm{mHz}$ can be derived from the hyperfine data found in Ref. 14]. It has a high insensitivity to external electromagnetic fields. The light-shift cancellation is expected to occur near $800 \mathrm{~nm}$, a wavelength far from any atomic resonance and for which powerful and practical laser sources are readily available. Apart from a residual effect due to hyperfine structure, the light shift cancellation is independent on polarization. Finally, higher order effects due to the trapping field are expected to be extremely small on this particular line 15]. In this Letter we report the first direct observation and frequency measurement of this transition.

The experiment is performed on a sample of cold atoms collected in a magneto-optical trap (MOT). An atomic beam is decelerated in a Zeeman slower and captured at the crossing-point of three retro-reflected beams tuned 40 MHz to the red of the ${ }^{1} S_{0}-{ }^{1} P_{1}(F=9 / 2)$ transition (Fig. 11). The setup is described in details in Ref. [16]. For this particular experiment $3 \times 10^{6}{ }^{87} \mathrm{Sr}$ atoms are trapped at steady state at a temperature of $2 \mathrm{mK}$. The atomic cloud has a $1 / e^{2}$ diameter of $2 \mathrm{~mm}$. To probe the $698 \mathrm{~nm}$ line, a $14 \mathrm{~mW}$ laser beam passes four times through the atomic cloud in a standing wave configuration. The probe beam waist radius is $1.3 \mathrm{~mm}$. At first sight, a direct detection of the very forbidden line with our system seems a desperate task: the resonance is expected to be Doppler broadened to $1.5 \mathrm{MHz}$ (FWHM) more than three orders of magnitude higher than the Rabi frequency $(0.8 \mathrm{kHz})$. Thus only $10^{3}$ atoms are expected to be resonant with the laser at a time. In addition, an efficient fluorescence detection of atoms in the ${ }^{3} P_{0}$ state is made difficult by the absence of cycling transition from this state. Finally the energy of the ${ }^{3} P_{0}$ state is known from databases to within a few hundred $\mathrm{MHz}$ only.

To overcome these problems we have first determined the resonance frequency with an accuracy of $110 \mathrm{kHz}$ by measuring the frequency of the ${ }^{1} S_{0}-{ }^{3} P_{1}$ line at $689 \mathrm{~nm}$ and of the frequency difference between the ${ }^{3} P_{1}-{ }^{3} S_{1}$ and ${ }^{3} P_{0}-{ }^{3} S_{1}$ transitions at $688 \mathrm{~nm}$ and $679 \mathrm{~nm}$ respectively (see Fig 1) 17]. Second, to amplify the fraction of cold 
atoms transferred to the ${ }^{3} P_{0}$ state by direct excitation, we have taken advantage of the fact that the lifetime of the MOT is 40 times larger than the Rabi oscillation period. A laser tuned to resonance induces a leak in the MOT leading to a detectable decrease of the number of trapped atoms of $1 \%$.

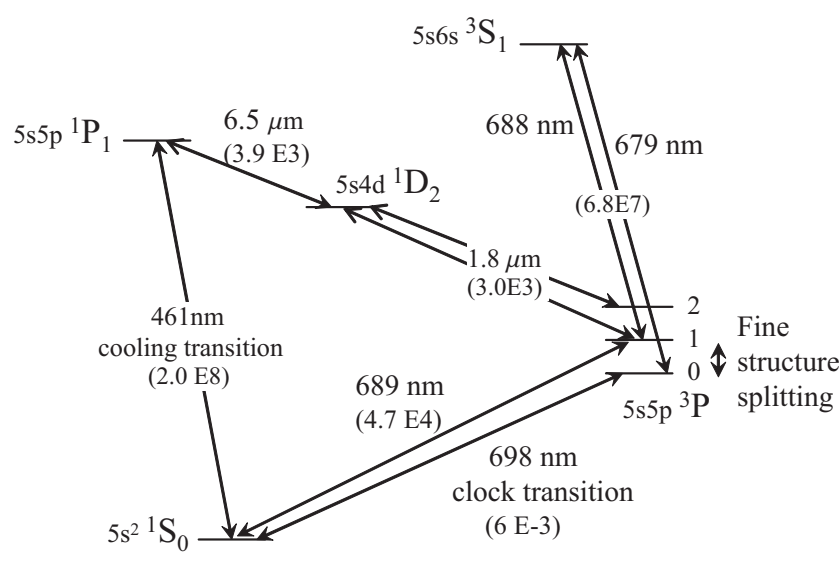

FIG. 1: Energy diagram of ${ }^{87} \mathrm{Sr}$ with wavelength and decay rate $\left(\mathrm{s}^{-1}\right)$ of the transitions involved in the experiment. For clarity, the hyperfine structure is not represented $(I=9 / 2)$.

The experimental set up used for all frequency measurements is described in Fig. 2 An extended cavity laser diode (ECLD1) is locked to a high finesse cavity using the Pound-Drever-Hall method 18] with performances reported in Ref. [19]. Its frequency is continuously measured vs a hydrogen maser with a scheme based on a self referenced femtosecond Ti:Sapph laser [20, 21]. The relative resolution of this measurement is typically $3 \times 10^{-13}$ for a one second averaging time. A second laser (ECLD2) is offset-phase locked to ECLD1. The beat-note between both lasers is mixed with the output of a radio frequency synthesizer to generate the offset phase lock error signal. The bandwidth of the servo control is $2 \mathrm{MHz}$. With this scheme the light of ECLD2, which is sent to the atoms, can be tuned to any frequency between two modes of the cavity of free spectral range $1.5 \mathrm{GHz}$ by actuating the RF synthesizer. Two sets of lasers are used. One can be tuned from 675 to $685 \mathrm{~nm}$, the other one from 685 to 698 nm.

The frequency of the ${ }^{1} S_{0}-{ }^{3} P_{1}(F=9 / 2)$ transition is measured with an atomic beam independent from the cold atom setup. The obtained frequency is 434829342 $950(100) \mathrm{kHz}$, with an uncertainty mainly dominated by statistical noise and imperfect knowledge of the magnetic field.

The determination of the fine structure splitting between ${ }^{3} P_{0}$ and ${ }^{3} P_{1}$ is performed with the cold atoms. While trapped and cycling on the ${ }^{1} S_{0}-{ }^{1} P_{1}$ transition, atoms eventually emit a spontaneous photon which brings them to the ${ }^{1} D_{2}$ state (Fig. 1). This state has two

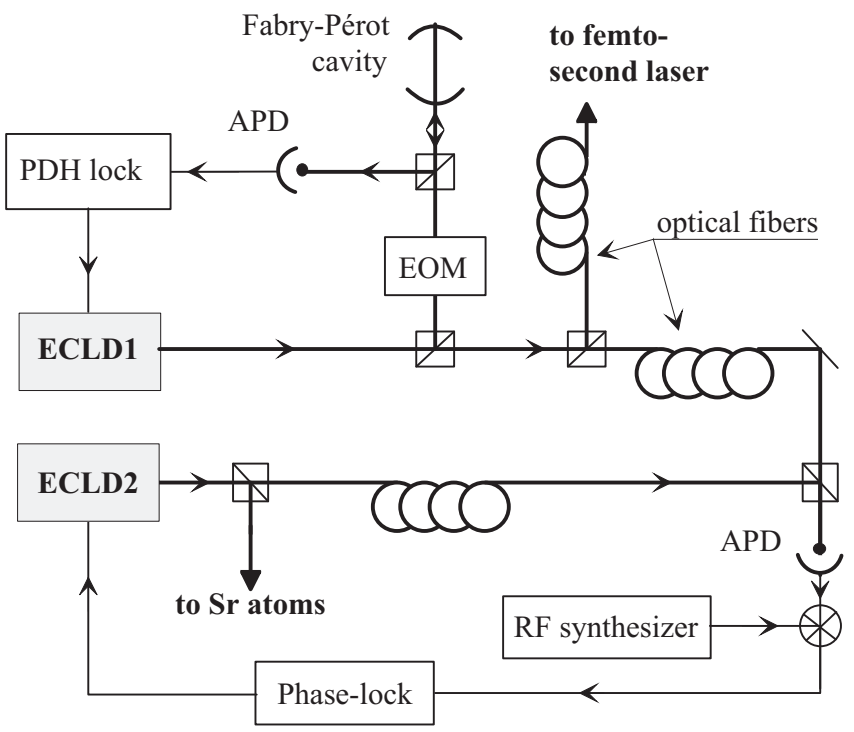

FIG. 2: Experimental set up used for frequency measurements. An extended cavity laser diode (ECLD1) is locked to a high finesse Fabry-Pérot cavity $(\mathrm{F}=27000) .100 \mu \mathrm{W}$ are sent to a frequency chain based on a femtosecond laser for absolute frequency measurement. A second laser (ECLD2) which is used to probe the strontium atoms is offset-phase locked to ECDL1. The beat-note collected by an avalanche photodiode (APD) is mixed with a radio frequency reference. Noise and frequency offsets introduced by optical fibers are negligible in this experiment. EOM: electro-optical modulator.

main decay channels: to the ${ }^{3} P_{1}$ and ${ }^{3} P_{2}$ states. Atoms in the ${ }^{3} P_{1}$ state decay back to the ground state and are kept in the trap while atoms in the metastable ${ }^{3} P_{2}$ state are lost. This process limits the lifetime of the MOT to some $50 \mathrm{~ms}$. We make the fine structure measurement by modifying this escape process. If a laser resonant to one of the hyperfine components of the ${ }^{3} P_{1}-{ }^{3} S_{1}$ transition is added to the trap, atoms in the corresponding ${ }^{3} P_{1}$ state are pumped to the ${ }^{3} P_{2}$ and ${ }^{3} P_{0}$ metastable states. They escape the trap instead of decaying back to the ground state. This decreases the trapped atom number. Fig. 3(a) shows the fluorescence of the MOT as a function of the $688 \mathrm{~nm}$ laser detuning from the ${ }^{3} P_{1}, F=9 / 2-{ }^{3} S_{1}, F=11 / 2$ resonance.

A direct detection of the ${ }^{3} P_{0}-{ }^{3} S_{1}$ transition by the same technique is not possible because ${ }^{3} P_{0}$ is not populated in the MOT. Instead, a first signal is obtained by detecting the light shift of the ${ }^{3} P_{1}-{ }^{3} S_{1}$ transition induced by a $679 \mathrm{~nm}$ laser close to the ${ }^{3} P_{0}-{ }^{3} S_{1}$ resonance. With an intensity of $1.8 \mathrm{~mW} / \mathrm{mm}^{2}$, a shift of $100 \mathrm{kHz}$ is observed for a detuning of $100 \mathrm{MHz}$. For a $679 \mathrm{~nm}$ detuning smaller than the width of the $688 \mathrm{~nm}$ resonance, a dip appears in the resonance profile due to coherent population trapping $(\mathrm{CPT})$ : when the frequency difference between both lasers matches the atomic fine structure, there exists a coherent superposition of ${ }^{3} P_{1}$ and 
${ }^{3} P_{0}$ states which is not coupled to ${ }^{3} S_{1}$ [22]. Atoms in this dark state are not pumped to ${ }^{3} P_{2}$ but decay back to the ground state in a few $10 \mu$ s due to the ${ }^{3} P_{1}$ instability. They are kept in the MOT. When the CPT dip is centered on the $688 \mathrm{~nm}$ resonance, the $679 \mathrm{~nm}$ laser is tuned to resonance. The observed signal in this configuration is shown in Fig. 3(b). The fine structure measurement is performed light shift free with both lasers locked on resonance. We measured a frequency of 5601338650 (50) $\mathrm{kHz}$ with an uncertainty mainly due to the magnetic field gradient of the MOT. According to these measurements, the ${ }^{1} S_{0}-{ }^{3} P_{0}$ transition is expected to have a frequency of 429228004300 (110) kHz.

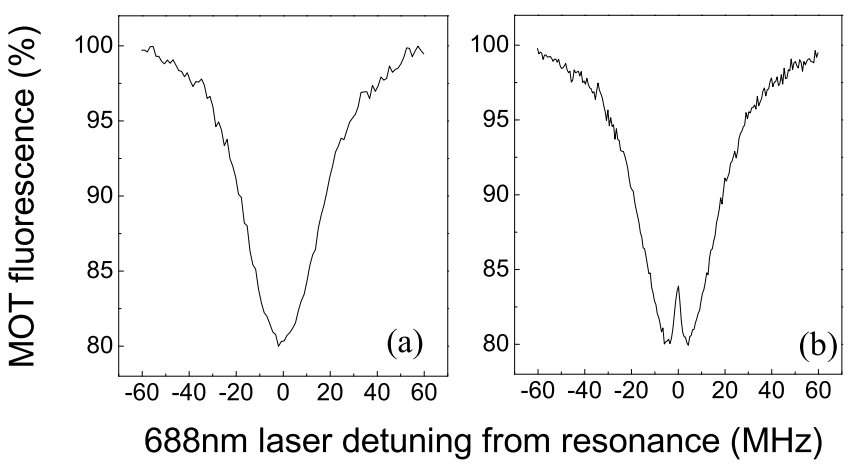

FIG. 3: Relative MOT fluorescence signals obtained when a $688 \mathrm{~nm}$ laser is swept around the ${ }^{3} P_{1}, F=9 / 2-{ }^{3} S_{1}, F=$ $11 / 2$ transition. (a) $688 \mathrm{~nm}$ laser only; (b) with an additional $679 \mathrm{~nm}$ laser tuned to the ${ }^{3} P_{0}, F=9 / 2-{ }^{3} S_{1}, F=11 / 2$ resonance. In this case the CPT dip is observed. Intensity of the $688 \mathrm{~nm}$ and $679 \mathrm{~nm}$ laser are $5 \mu \mathrm{W} / \mathrm{mm}^{2}$ and $2 \mathrm{~mW} / \mathrm{mm}^{2}$ respectively.

For the direct observation, we induce in the MOT a leak to the ${ }^{3} P_{0}$ state with a laser tuned to resonance. The lifetime of the trap is two orders of magnitude longer than the duration of a $\pi$ pulse on the forbidden transition with the laser parameters given above. This leads to a build-up by the same factor of the fraction of atoms escaping the MOT if the transfer rate to ${ }^{3} P_{0}$ is constant and if the atoms, once in the ${ }^{3} P_{0}$ state, actually escape the trapping process. One way to fulfil the first condition could consist in using the MOT to rethermalize atoms to fill the dip in the velocity distribution created by excitation to ${ }^{3} P_{0}$. For the escape condition, one could think of optical pumping to ${ }^{3} P_{2}$ with a laser tuned to ${ }^{3} P_{0}-{ }^{3} S_{1}$. In our experiment however, the Doppler effect induced by gravity is sufficient to fulfil both conditions. It amounts to several times the Rabi frequency per millisecond with the $45^{\circ}$ angle formed by the $698 \mathrm{~nm}$ probe beam and vertical. The dip in the velocity distribution induced by the excitation laser is then permanently refilled. On the other hand, atoms in the ${ }^{3} P_{0}$ state are rapidly detuned from the excitation laser and do not emit a stimulated photon back to the ground state. The trapped atom number should then decrease by several $\%$.

In practice, the experiment is operated sequentially: by means of acousto-optic modulators we alternate a capture and cooling phase with the blue lasers and a probe phase with the $698 \mathrm{~nm}$ laser. The main motivation for this pulsed operation is to avoid any light shift of the forbidden transition. Another reason is related to the escape process of the ${ }^{3} P_{0}$ atoms. It would be less efficient with the MOT beams on due to the instability of the ${ }^{1} S_{0}$ state confered by the coupling to ${ }^{1} P_{1}$ : the reduced lifetime of the atomic coherences due to spontaneous emission of blue photons would lead to an effective broadening of the $698 \mathrm{~nm}$ excitation much larger than the Doppler effect due to gravity. The optimization of the time sequence results from a trade-off between the capture efficiency of the MOT, the ballistic expansion of the atomic cloud during probe phases, and the efficiency of excitation to ${ }^{3} P_{0}$. With phases of capture of $3 \mathrm{~ms} \mathrm{du}-$ ration and probe phases of $1 \mathrm{~ms}$ duration, we have $1 \times 10^{6}$ atoms at steady state in the MOT and the contrast of the resonance is $1 \%$. In figure 4 is shown the fluorescence of the trapped atoms versus the $698 \mathrm{~nm}$ laser detuning from resonance. A narrow sub-Doppler structure is expected at the center of the resonance due to the standing wave configuration. With the present signal to noise ratio, we are not able to detect it.

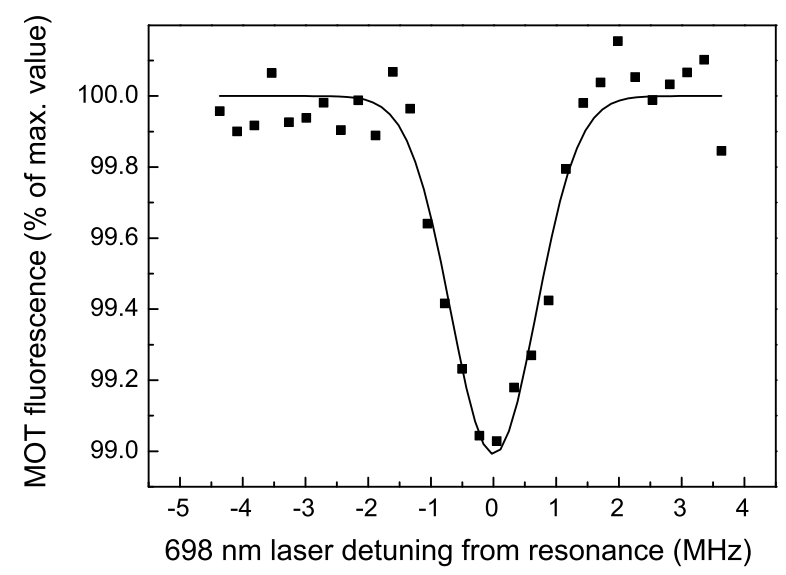

FIG. 4: Direct observation of ${ }^{1} S_{0}-{ }^{3} P_{0}$ transition. The line is broadened by the Doppler effect due to the finite temperature of the atoms to $1.4 \mathrm{MHz}$ (FWHM).

We have locked the laser to the ${ }^{1} S_{0}-{ }^{3} P_{0}$ resonance. Its frequency is $429228004235 \mathrm{kHz}$ with a standard deviation of $20 \mathrm{kHz}$ for an averaging time of 2 hours. At that level, the systematic effects are negligible. The first order Doppler effect vanishes due to the standing wave configuration. The Zeeman effect induced by the MOT field gradient is less than $1 \mathrm{kHz}$, since the Landé factors of the ${ }^{1} S_{0}$ and ${ }^{3} P_{0}$ levels are both equal to $-1.3 \times 10^{-4}$. The light shift which would result from imperfect extinction 
of the blue light is also estimated below $1 \mathrm{kHz}$. Tab. I summarizes the indirect and direct measurements and confirms the good agreement between both methods.

TABLE I: Measured frequencies of ${ }^{87} \mathrm{Sr}$ transitions $(F=9 / 2$ for all atomic states).

\begin{tabular}{l|c}
\hline \hline Transition & Frequency (kHz) \\
\hline \hline $5 s^{2}{ }^{1} S_{0}-5 s 5 p^{3} P_{1}$ & $434829342950 \pm 100$ \\
\hline $5 s 5 p^{3} P_{0}-5 s 5 p^{3} P_{1}$ & $5601338650 \pm 50$ \\
\hline $5 s^{2} S_{0}-5 s 5 p^{3} P_{0}$ \\
\hline indirect measurement & $429228004300 \pm 110$ \\
\hline direct measurement & $429228004235 \pm 20$ \\
\hline \hline
\end{tabular}

The technique used for the direct detection of this line can be extended to other atomic species. As an example, $\mathrm{Yb}$ has the same level scheme as $\mathrm{Sr}$ with several isotopes having non-zero nuclear spin. With $\mathrm{Yb}$ atoms trapped on the ${ }^{1} S_{0}-{ }^{3} P_{1}$ transition of natural width $200 \mathrm{kHz}$, the lifetime of the MOT can be much longer than the blue Sr MOT, up to several seconds in Ref. [24]. The contrast of the forbidden line would then be close to $100 \%$.

The observation of the ${ }^{1} S_{0}-{ }^{3} P_{0}$ transition is a first step towards the realization of an optical frequency standard using trapped neutral atoms. Clearly the next step is the measurement of the wavelength of the dipole trap beam where light-shift cancellation occurs. This measurement requires better frequency resolution than achieved here, i.e. higher laser intensity at $698 \mathrm{~nm}$ and/or colder atoms.

In this new type of optical frequency standards a line- $Q$ as high as $10^{15}$ is achievable if the spontaneous emission rate in the optical trap is less than one per second. With a laser intensity of $2 \times 10^{7} \mathrm{~W} / \mathrm{m}^{2}$, the trap oscillation frequency can be $50 \mathrm{kHz}$ and the spontaneous emission rate $0.6 \mathrm{~s}^{-1}$. With a line- $Q$ of $10^{15}$ and a reasonable trapped atom number of the order of $10^{6}$, ultimate performances are orders of magnitude better than existing devices. The frequency noise of the laser used to probe the atoms will then be of decisive importance [19]. State of the art ultra stable lasers use macroscopic resonators as a reference and exhibit $1 / f$ frequency noise at Fourier frequencies below a few $\mathrm{Hz}$ [23]. We propose to circumvent this problem with a first stage servo control to the atomic transition with a separate setup fully optimized for frequency stability: large number of atoms, favorable duty cycle... At the price of an increase of the experimental width of the atomic resonance the response time of the servo-control can be as fast as desired. With this additional degree of freedom, the optimization of ultrastable lasers should lead to a large improvement of their performances. One could then approach the demanding requirements of the optical standard using trapped neutral atoms.

We thank Ouali Acef, André Clairon, Michel Lours and Giorgio Santarelli for helpful discussions, and the optoelectronic group of the university of Bath (UK) for providing the photonic cristal fiber of the frequency chain. A. B. acknowledges his grant from the european Research Training Network CAUAC. BNM-SYRTE is Unité Associée au CNRS (UMR 8630).

* Electronic address: pierre.lemonde@obspm.fr

[1] H. Marion et al., submitted to Phys. Rev. Lett. (2003).

[2] S. Bize et al., submitted to Phys. Rev. Lett. (2003).

[3] T. Damour and F. Dyson, Nucl. Phys. B480, 37 (1996).

[4] J. Webb et al., Phys. Rev. Lett. 87, 091301 (2001).

[5] S. Bize et al., in Sixth Symposium on frequency standards and metrology, edited by P. Gill (World Scientific, Singapore, 2002).

[6] R. J. Rafac et al., Phys. Rev. Lett. 85, 2462 (2000).

[7] S. A. Diddams et al., Science 293, 825 (2001).

[8] M. Niering et al., Phys. Rev. Lett. 84, 5496 (2000).

[9] F. Riehle, G. Wilpers, T. Binnewies, and J. Helmcke, in Sixth Symposium on frequency standards and metrology, edited by P. Gill (World Scientific, Singapore, 2002).

[10] E. A. Curtis et al., in Sixth Symposium on frequency standards and metrology, edited by P. Gill (World Scientific, Singapore, 2002).

[11] J. Stenger et al., Opt. Lett. 26, 1589 (2001).

[12] See Sixth Symposium on frequency standards and metrology, edited by P. Gill (World Scientific, Singapore, 2002).

[13] H. Katori, in Sixth Symposium on frequency standards and metrology, edited by P. Gill (World Scientific, Singapore, 2002).

[14] H.-J. Kluge and H. Sauter, Z. Phys. 270, 295 (1974).

[15] V.G. Pal'chikov, J. Opt.B 5, in press (2003).

[16] I. Courtillot et al., Opt. Lett. 28, 468 (2003).

[17] We also performed absolute frequency measurements of ${ }^{1} S_{0}-{ }^{3} P_{1},{ }^{3} P_{0}-{ }^{3} S_{1}$ and ${ }^{3} P_{1}-{ }^{3} S_{1}$ transitions for all hyperfine sublevels of ${ }^{87} \mathrm{Sr}$ and ${ }^{88} \mathrm{Sr}$. These measurements will be reported subsequently.

[18] R. W. P. Drever et al., App. Phys. B 31, 97 (1983).

[19] A. Quessada et al., J. Opt.B 5, in press (2003).

[20] R. Holzwarth et al., Phys. Rev. Lett. 85, 2264 (2000).

[21] D. J. Jones et al., Science 288, 635 (2000).

[22] G. Alzetta, A. Gozzini, L. Moi, and G. Orriols, Nuovo Cimento B36, 5 (1976).

[23] B. Young, F. Cruz, W. Itano and J. C. Bergquist, Phys. Rev. Lett. 82, 3799 (1999).

[24] T. Kuwamoto, K. Honda, Y. Takahashi, and T. Yubuzaki, Phys. Rev. A 60, R745 (1999). 